\title{
The Cultural Heritage Open Laboratory System (CHeLabS). Unfolding a cultural heritage-driven development in science and technology
}

\author{
Paola Calicchia ${ }^{1}$, Luca Pitolli ${ }^{2}$, Paolo Salonia ${ }^{3}$ \\ ${ }^{1}$ Institute of Acoustics and Sensors "O. M. Corbino"/National Research Council, Via Fosso del Cavaliere 100, 00133 Rome, Italy \\ ${ }^{2}$ Institute of Complex Systems/National Research Council, Via dei Taurini 19, 00185 Rome, Italy \\ ${ }^{3}$ Institute for Technologies Applied to Cultural Heritage/National Research Council, Via Salaria Km. 29,300 - C.P. 10,00015 Monterotondo St. \\ (Rome), Italy - ICOMOS IT Executive Board
}

\begin{abstract}
A territory, with its distinctive cultural heritage ecosystem effectively linked to the technological capabilities, represents a co-evolved system that contributes to the socio-economic growth and to a knowledge-based society.

This work illustrates a new model of territorial system recently launched, the Cultural Heritage Open Laboratory System (CHeLabS), that aims at the construction of a favourable context addressing the challenge of a $\mathrm{CH}$-driven development in science and technology. The concept, the inspiring principles, the vision, the activities and the implementation steps of this system are discussed, in addition to some key factors that trigger innovation and sustain knowledge building dynamics useful for shaping the future CHeLabS environment. The cultural asset is at the centre of this system, where a number of representative heritage sites and monuments are the nodes of a scalable and distributed laboratory, based on the Open Access and Sharing culture.

The current and bottom-up phase of implementation of the system is described: on a web-based platform, the heritage science community is invited to participate to an interactive survey. The expected results will highlight the problems, perceived by the experts community, demanding for new knowledge and also the technological trends that could unfold effective innovation dynamics.
\end{abstract}

Section: RESEARCH PAPER

Keywords: cultural heritage territorial system; monuments and sites safeguard; multidisciplinary innovation dynamics; open access\&sharing

Citation: Paola Calicchia, Luca Pitolli, Paolo Salonia, The Cultural Heritage Open Laboratory System (CHeLabS). Unfolding a cultural heritage-driven development in science and technology, Acta IMEKO, vol. 6, no. 3, article 2, September 2017, identifier: IMEKO-ACTA-06 (2017)-04-02

Section Editor: Sabrina Grassini, Politecnico di Torino, Italy

Received March 14, 2017; In final form March 14, 2017; Published September 2017

Copyright: @ 2017 IMEKO. This is an open-access article distributed under the terms of the Creative Commons Attribution 3.0 License, which permits unrestricted use, distribution, and reproduction in any medium, provided the original author and source are credited

Funding: This work was supported by National Research Council within the Project SM@RTINFRA - SSHCH (MIUR MD n. 973 of 25.11.2013)

Corresponding author: Paola Calicchia, e-mail: paola.calicchia@idasc.cnr.it

\section{INTRODUCTION}

The present work fundamentally wishes to conjugate the basic safeguard principles, consolidated both in the theoretical speculations and in the practices, with the identification of innovative technologies and their sustainable development. While the effective preventive conservation represents the objective towards which this integration is oriented, the proposed approach deals with the building of a system that connects the heritage assets, the conservation operators, the research facilities, and the local communities.

On one side, the first relevant assumption of the existence of this kind of dialogue can be found in the conservation discipline expressed in The Venice Charter in 1964 [1] (following which the International Council of Monuments and Sites - ICOMOS was established [2]) stating, in art. 2, that "The conservation and restoration of monuments must have recourse to all the sciences and techniques which can contribute to the study and safeguarding of the architectural heritage". Thus, it introduces the concept of the diffusion of the scientific and technical knowledge into the safeguard practice for those heritage assets that, due to their intrinsic value, represent the World Heritage.

The Venice Charter opened the way to a number of 
important recommendations for the specific cultural heritage conservation domain, proposed by public and private organizations, among which ICOMOS as UNESCO advisory body. From these documents it is possible to get some meaningful indications to which the project, presented in this paper, was inspired. Mainly, we wish to refer to the Nara Document on Authenticity (1994) [3], to the Faro Convention (2005) by the Council of Europe on the value of cultural heritage for society [4], to the Hangzhou Declaration (2013) that sets the culture at the center of the policies for the sustainable development [5], leading to the UNESCO Agenda 2030 and the Sustainable Development Goals (SDGs) [6].

Mentioning the final document of the ICOMOS General Assembly in Florence (2014), we can recall the importance of the so called community-driven practices, and the capacity building, as well as the recommendations for an informed use of the innovative technologies (Florence Declaration Heritage and Landscape as Human Values) [7].

Finally, the premise of the Guidelines to the Annex of the UNESCO Convention on the Protection of the Underwater Cultural Heritage (2001) [8] suggests a paradigm shift in the motivation of the onsite preservation, built on the awareness that "Heritage sites are not an inexhaustible resource. [...] The future bolds unimaginable advances in technology, which may lead to far more innovative methods of trace analysis that could profitably be used in archaeology. Even more importantly, research questions develop step-bystep, building on prior knowledge and understanding. In order to address research questions that will arise in the future as a result of this creative scientific process, it is essential that at least a selection of sites remains untouched and researchable." Adopting this perspective not only for underwater heritage, the heritage sites are also perceived as a space whose onsite preservation has a value for the experimentation and for the scientific and technological innovation, with the awareness that the scientific knowledge creation process will profitably contribute to the future preservation practices.

Considering now the other element of the initial dialogue related to the scientific facilities, the networks and the research infrastructures, specifically those of the hard science applied to the heritage domain, it is worth noted the vision of the ESFRI Strategy, as part of the most advanced policy in building systems [9]. Firstly the CHARISMA EU project, and presently the IPERION-CH EU project have strongly pushed the culture of the open access facilities among this community. This recalls the well known experience of all the large-scale facilities that, in few decades, have advanced the knowledge in many research fields and brought a positive growth on the hosting territory. Furthermore, a modern environmental planning and management approach for cultural heritage sites was developed in the CHERPLAN project, within the SEE program [10]. This approach provided a strong base for a synergy between conservation and socio-economic growth of the territory (principles of Agenda UNESCO 2030).

Concerning long-term actions addressing innovation in the cultural heritage safeguard, the experience of few decades of hard science applications in this field suggests the need of both the following aspects: i) an insight into single innovative technologies and their opportunities; ii) a better understanding of the favourable context and the collective dynamics characterizing the behaviour of the heritage community with respect to innovations.

In our vision, the innovation dynamics based on knowledge creation is an essentially collective process, occurring inside a community acting as a group or, more precisely, as a complex adaptive system. The environment and the quality of the interaction within the community may effectively orient this process. We refer here to some basic concepts derived from the science of complex systems. Specifically, for this task, we refer to the studies conducted on the dynamics inside the highperformance working teams [11], extensively applying the concept of working team to larger groups of scholars focused on the same topic. Furthermore, attention is paid to a rather new research field studying the dynamics of creativity and innovations [12], [13], for a better understanding of the behaviour of both individuals and communities exposed to innovations.

This work presents the vision statement of the Cultural Heritage Open Laboratory System (CHeLabS), and describes its fundamental characteristics for addressing innovation in the heritage domain. This is a new model of territorial system, still in its initial phase of development, where a number of representative heritage sites and monuments are the interconnected nodes of a scalable and distributed laboratory, based on the Open Access and Sharing culture. The novelty of the CHeLabS system resides in the combination of the open access policy, for strengthening innovation potential in the heritage science, with the recognition that the cultural asset, grounded in the territory, offers the scenario for a new territorial growth.

The concept of the CHeLabS system is presented in Section 2 , and its subsections, including a description of: the inspiring principles in 2.1; the vision in 2.2; the potential activities in 2.3; the implementation steps in 2.4. Successively, the current phase consisting in the implementation of the CHeLabS platform, is described in Section 3, and its subsections, encompassing the description of: the community in 3.1; the survey in 3.2; the interactive map in 3.3. Finally, some general aspects are summarized in the conclusions, in Section 4.

\section{THE CHELABS SYSTEM}

Each element belonging to the cultural heritage is usually exposed to a particular risk, is characterized by a specific decay process evolution, requires defined risk management plans and conservation actions. Although economic barriers may complicate the adoption of good practices, in many cases the most critical issues can be faced through the application of known tools, fully validated methodologies and well assessed approaches. In other cases effective tools and solutions to these critical issues may not be already known, or mature enough to be available on site at the proper time to avoid impairs to those assets that need to be preserved. Thus unsolved problems can be identified and challenging issues characterize these heritage sites. Actually, they are the most interesting and adequate sites within the CHeLabS vision because they definitely lay at the frontier of what is already known, while demanding for new knowledge. These cases show an added value: they offer an attracting context able to solicit the experts community to move a step forward, while effective solutions are not yet apparent.

The CHeLabS approach recognizes in these contexts the opportunity to foster the scientific and technological knowledge, contributing to their attractiveness, though they need to gather other relevant key factors for triggering innovation in the cultural heritage domain, as discussed later on. 


\subsection{Inspiring principles}

In many fields of science as in everyday life the comprehension of the dynamics of innovation is essential for facing challenges and exploring new solutions to unsolved problems.

In the domain of social psychological science, also related to the economic science, many studies investigated the distinctive characters of the high performance working teams. High performance working teams, when observed during meetings and analysed in terms of cross-correlations of speech acts, were found to be characterized by chaotic dynamics [11]. Chaotic dynamics is related to the behaviour of dynamical systems that are highly sensitive to initial conditions. So that very little differences in initial conditions lead to very different evolutions, making impossible any long-term prediction. Beyond unpredictability the positive aspect is that, chaotic dynamics is linked to the optimal learning landscape and to adaptation (i.e. the ability to respond flexibly to a changing environment), as well as to a high degree of connectivity of the team.

Other studies investigated the role of leadership facilitating exploratory behaviours among the members of a team [14], [15]. In this framework, the leadership plays a fundamental role in shaping the processes within the team by cultivating openness, effective group interaction, exchange of ideas and a non-evaluative context during this exchange. It is also evidenced the need for the members of the group to effectively process and integrate the shared information, as well as the importance to have the opportunity or motivation to build on the ideas of others [15]. In the CHeLabS vision these concepts could be extended to a community of experts, acting as a group within the shared context of an open heritage site: tools, platforms, policies and a proper management enhancing the connectivity, and encouraging the knowledge sharing and integration among the community, are key factors of a favourable environment.

The heritage science domain has an added value in the multidisciplinary. Within the CHeLabS vision, each subject of the community has an offer and a need. Any need, evidenced by one subject, can be satisfied by the offer brought by another subject. Any participant can share his offer and explicit his need in a peer process, which can be here identified with the term reciprocity. Amplifying the scope of the concept cited above, we dare say that each subject may experience the importance of having the opportunity or the motivation to explore on the base of the need/offer of others.

The cited research [15] also recalls some threats that reduce the positive dynamics, bringing the groups to converge towards the direction of low performers. One of these factors is the attitude to focus on information which the members have in common, instead of taking advantage of the unique expertise of each member and benefit of such diversity. This threat is very frequent inside multidisciplinary contexts, such as in the heritage science domain, encompassing terminology and scientific background among humanities and hard science. In our vision, a fundamental key factor in the multidisciplinary environments is the attitude to explore the diversity, preventing the confinement into what is in common.

The free access to datasets and previous knowledge, the experimentation facing unsolved problems but also the meeting of constraints, and the compliance to stringent practical requirements push the whole community towards innovative solutions. Besides innovative events are usually correlated to, or triggered by, a previous novelty or innovation. In the domain of the science of complex systems, recent projects investigate the dynamics of creativity, novelties and innovation. Mathematical models are purposely analysed for mapping and predicting the occurrence of innovative events within an environment, and the correlation between successive events, in order to understand which is the optimal landscape for unfold creativity and innovation [12]. The research actually encompasses both social, biological and technological systems.

These studies are based on the interesting Kauffman's theoretical concept of the so called adjacent possible. Loreto et al. [13] describes this notion as follows "Originally introduced in the framework of biology, the adjacent possible metaphor include all those things, ideas, linguistic structures, concepts, molecules, genomes, technological artifacts, etc., that are one step away from what actually exists, and hence can arise from incremental modifications and/or recombination of existing material. [...] The strange and beautiful truth about the adjacent possible is that its boundaries grow as one explores them. The very definition of adjacent possible encodes the dichotomy between the actual and the possible [...] the actual realization of a given phenomenon and the space of possibilities still unexplored'. Furthermore, the cited authors suggest that this correlation of innovative events is realized by exploration of a space physical, conceptual, technological or biological - that enlarges itself as a new region is reached. From this perspective we can think that this space needs to be populated with existing materials, and in order to be populated (things and people) this space needs to be shared, where the accessibility and the connectivity between existing things and people is sustained. In the CHeLabS vision a shared space, where the exposure of the experts community to the unsolved problems and to the challenging issues is supported, becomes a space of possibilities where any new piece of knowledge opens up the opportunity to reach other regions of knowledge still unexplored.

The studies on these topics are still young but the expected outcomes regard some general rules for the identification of the determinants of creativity, and of the schemes for modelling the dynamics of innovations. Furthermore great expectation is in finding, within different contexts, the key factors that enhance creativity, as well as the optimal environment for it. In our vision, the above mentioned space is inside or around the object of heritage interest. Specifically the beritage asset, when exposed to its characteristic decay process, is considered the observable system that can be monitored and analysed in its natural evolution. When top-level expertise and state of the art technologies are gathered in this space, a new scenario will be opened: the cultural asset becomes the space where methodological and technological innovation systematically occur. We also suggest that in the heritage domain, where a great number of methodologies and technologies have been introduced in few decades, it becomes important to have a sort of scalable ability to see in depth to single new technologies and to see extensively to the dynamics of the community integrating these innovations. It is fundamental to observe how the multidisciplinary community of the heritage science behaves in its distinctive environment; under this perspective, next to the heritage asset, the community itself becomes the other observable system to study.

The main categories and the related key factors, introduced and described in this section, are synthesized in the scheme shown in Figure 1. 


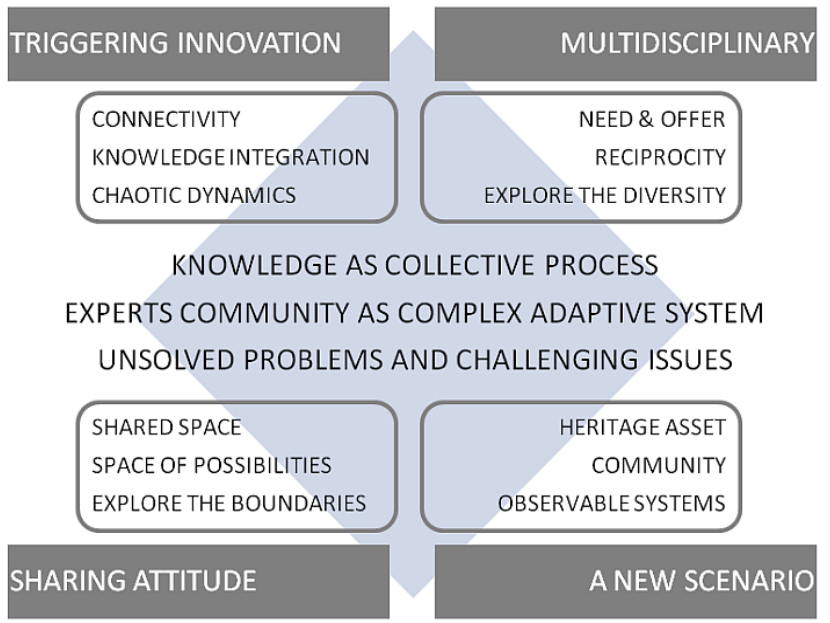

Figure 1. Scheme recalling the inspiring principles of the CHeLabS system: basic assumptions, main categories and relevant key factors.

\subsection{Vision}

According to the previous consideration, in the CHeLabS vision sites and monuments, historical centres and collections are at the centre of the system, becoming the places of a dynamic integration among people, different expertise and capabilities. This process is possible by implementing the access policies, the management, the most advanced technologies, and the activities encompassing research, training and dissemination actions within the patrimony itself. As shown in Figure 2, all these aspects are defined during the implementation phase (on the left of the scheme) and are useful to configure the services to the external users, and to accomplish their activities during the operative phase (on the right of the scheme).

In the cultural heritage domain, any relevant project includes a pilot site or a number of case studies, in order to conduct field experimentation of innovative systems or of new approaches in conservation science. Of course the on-site experimentation is accessible only to the project partnership. This practice guaranties that the planned activity can be properly accomplished following defined workflows; nevertheless it can be observed that this kind of context can also confine the potential for a more extensive knowledge integration. Once a heritage site is equipped with the most advanced technological instrumentation for monitoring and diagnostics, a suitable policy regulating the access of external experts can amplify the

IMPLEMENTATION

SERVICES

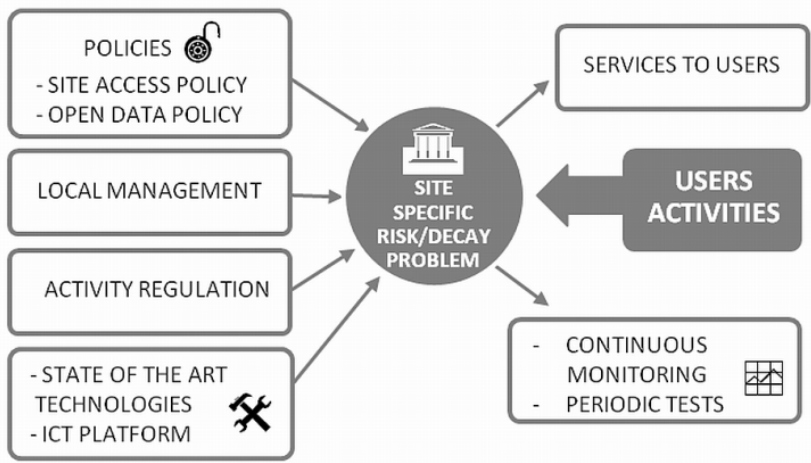

Figure 2. Configuration of a site of heritage interest when integrated into the CHeLabS system. scope and scale of the knowledge building process.

In the CHeLabS system, the implementation of a site access policy is envisioned to open a heritage site to external users, among the experts community, who wish to carry out their own research activity. This well-known approach, commonly adopted in many world-class facilities and open access laboratories (OL), is regulated through periodical calls for proposal and shows the advantage of widening the variety of expertise gathered up for studying the same topics.

Nowadays open access to information resources and datasets (OD) is one of the most important key factors for innovation, and generally recognized as a global trend. The free access to OD is sustained by many international organizations, among which UNESCO stating "While open information resources are main part of the knowledge generation, dissemination and growth cycle, open data will help in new interpretations, trend predictions and diverse and innovative applications of data" [16]. While in the past the data were held confidential for producing profit, today the awareness is growing to recognize the OD as a tool for producing new knowledge. Thus a paradigm shift is occurring today, moving from the enhancement of goods production towards the advancement of knowledge. The implementation of the OD policy represents a fundamental element for the CHeLabS system, also considered as an empowerment of the site access policy. While inducing the users to be engaged into a collective process, the availability of OD helps the integration of heterogeneous pieces of knowledge more than their sum.

One key factor for building creative contexts is supporting the sharing attitude of a community. This attitude shall be addressed in the policies and may encompass different aspects: i) sharing the place of the heritage sites, where different expertise may convene and work on a same problem; ii) sharing the state of the art technologies contributes to the capacity building process; iii) sharing knowledge helps the integration of information and the creation of new knowledge, of new solutions to unsolved problems, of new products or services; iv) sharing scientific and traditional knowledge by integrating the experts community and the local community. In this way the sharing attitude is strictly related to the concept of sustainability, including equal opportunities in the scientific advancement and in the sustainable development.

Beyond the access policies, for a heritage site to become a node of the CHeLabS system it is essential to create a local team for the site management (Local Unit), to make the most advanced instrumentations available onsite for continuous monitoring and periodic tests, as well as an ICT platform. The local units have the responsibility to define and regulate a list of possible activities that can be proposed and realized in that specific site, as well as a defined period for the open access policy according to the needs for conservation actions (also in terms of enhancement and avoiding the interruption in the enjoyment of goods).

When a number of sites, configured in such a way, are linked together they constitute a distributed laboratory, with a general management involved into a harmonization process.

The envisioned CHeLabS structure, as shown in Figure 3, accounts for a Coordinating Team, a Sites Management composed of the Leaders of the Local Units, and these last involved in the management of each single site. An Advisory Board is appointed to give support for defining the goals and the policies, the harmonization of the sites, the best practices exchange, the standardization of methods, the quality assurance, and the validation of shared data. The system of sites, with their 


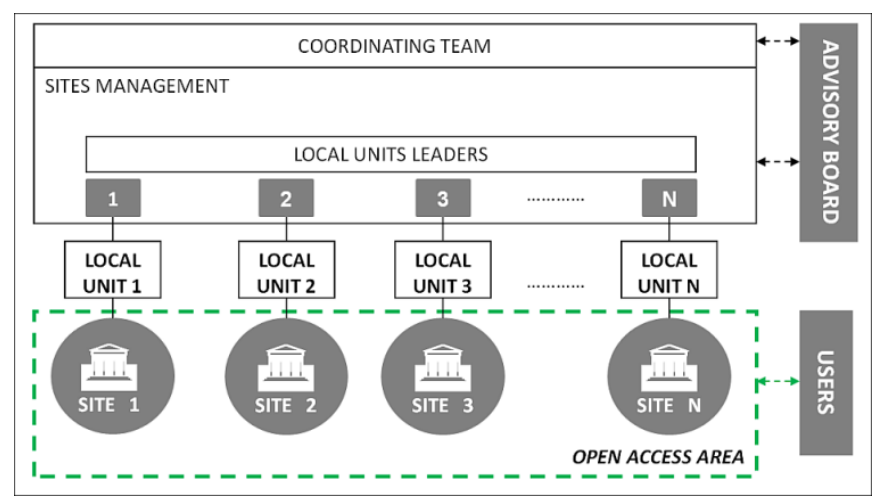

Figure 3. Structure of the CHeLabS system.

distinctive character, constitutes an Open Access Area accessible, for on-site investigations, to the local units and to the external users who propose their activities. This system is a scalable and harmonized network involving different players in a multidisciplinary context, stimulating those dynamics that trigger events favourable to creativity and innovation.

\subsection{Potential activities}

On a CHeLabS site, the Local Unit together with the organization, responsible for the safeguard of the heritage site, define the allowed activities and the requirements useful to regulate the accessibility. The users can access: the beritage asset, the state of the art instrumentations, the information and datasets.

According to the above regulation, the possible activities can be organized as follows:

KNOWLEDGE: research on the heritage asset using the instrumentations available onsite;

DIAGNOSTICS/MONITORING: research on the risk/decay kinetics using the instrumentations available onsite;

TECHNOLOGY: research on the instrumentations brought by the users, or available onsite, used to study the heritage asset;

COMPARISON: research on the users' instrumentation, applied to the heritage asset, by means of a comparative analysis using the instrumentations available onsite;

VALIDATION: validation of innovative technologies or analytical methods developed by the users, employed on the heritage asset, by integrating the results of the onsite experimentation with the information from previous knowledge and datasets about the heritage asset;

PROTOTYPE TESTING: a prototype of an innovative device or instrumental system becomes part of the instrumentations available onsite, in order to be tested by a great number of users and obtain information on its applicability and potential improvements;

TRAINING: professional courses including theory and practice, focused on the study of the heritage asset or on the instrumentations available onsite.

Beyond this variety of activities that can be realized on a single site, more articulated actions can be easily accomplished in the CHeLabS system as a system of sites. For instance:

PRACTICES EXCHANGE: if two or more sites of the system present similar elements, a successful practice developed in one of them can be easily verified on the other ones by simple internal bureaucratic procedures.

This possibility accelerates the process for the verification and the adoption of good practices, according to the standards established by the authoritative organisations.
APPLICABILITY: if a user needs to undertake an applicability assessment of an innovative method/device by testing it on different artifacts, he can propose to apply the same experimental method on different heritage assets available on different sites. For instance an innovative diagnostics tool, after laboratory validation, may need to be verified on different types of paintings. The CHeLabS system can guarantee the accessibility to a suitable variety of paintings in a suitable period of time, allowing the user to easily accomplish this task.

This possibility accelerates the time-to-market of new important technologies with a solid background of experimental tests, and their adoption within the conservation practices.

PROTOCOL-STANDARD: as for the good practice exchange, also protocols and new standards can be implemented on a knowledge base, built by integrating the results of the experimentation on different sites.

This possibility helps the harmonization and the standardization processes.

All the above mentioned activities involve many aspects, relevant for a solid approach based on those key principles belonging to the metrology framework. For instance, the validation of methods, the use of comparison, the calibration of the instruments available onsite, the use of reference materials, and finally the implementation of standards.

\subsection{Implementation steps}

What happens when a site of significant heritage interest becomes a node of the CHeLabS system?

Let us analyze a practical example where the implementation of the CHeLabS system is accomplished in an archaeological area. Let us assume that this area is located in the proximity of a lagoon, and is characterized by seismic hazard related to the geomorphology of the soil and by flooding. Furthermore, we can also assume that structural decay processes affect the built heritage located in that area. This site may present a number of challenges in the comprehension of the geophysical characteristics of the territory, correlated to the accurate knowledge of the structural decay evolution in the buildings, and in the risk management; all these challenging issues require the development of innovative approaches as well as technologies for effective and preventive conservation actions.

CHeLabS integrates the site into the system, through the following phases, synthetically shown in Figure 4:

PHASE 1 - Candidature of the site

The organization responsible for the safeguard of the archaeological area candidates the site to the CHeLabS system, and agrees to adopt the proper policies for the accessibility of the site and the data regarding the site. The management identifies the specific requirements in order to regulate the possible users activities, such as:

Allowed activities - research studies; validation; comparison; training camp; dissemination events;

Annual availability - maximum six months per year;

Requirements - Compatibility with visitors access and enjoyment;

Additional spaces for storing external users' equipments available onsite;

Duration of the open access policy - five years.

PHASE 2 - Evaluation of the candidature

The CHeLabS Advisory Board evaluates the application analyzing the representativeness of the site with respect to the risk/decay specificity, and identifies challenging issues that are 


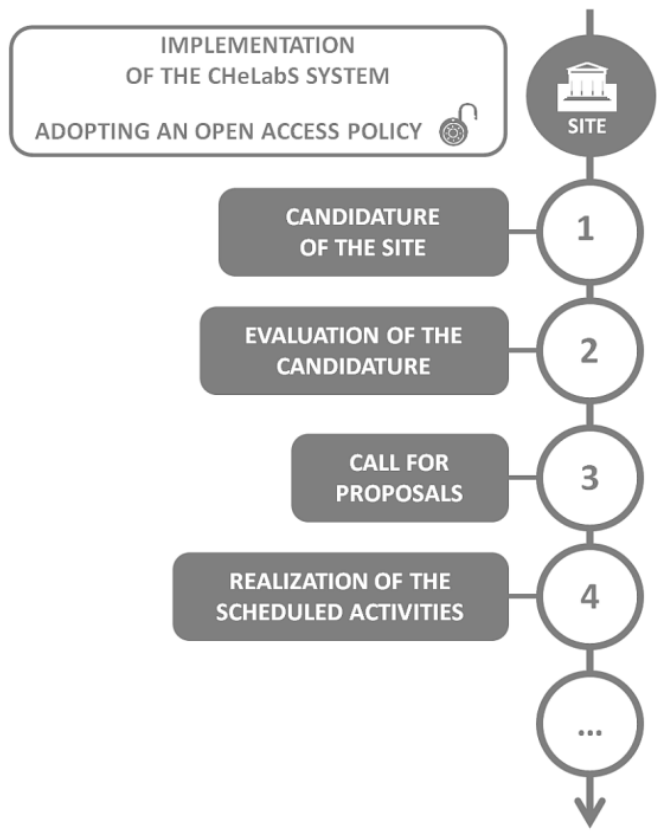

Figure 4. Fundamental steps constituting the preparatory phase, aiming at the integration of a heritage site into the CHeLabS system.

strategic elements for the accomplishment of the CHeLabS objectives. All the practical aspects related to the authorization are preventively faced and solved at this stage. Successively, in order to prepare the site for the full operability, the equipment of the site is accomplished with the state of the art instrumentations which are now available onsite, such as:

Devices for specific studies - multichannel seismic antennas; systems for geophysical measurements of the subsoil; systems for structural tests for the static deformation parameters definition in buildings.

Devices for constant monitoring - seismic sensors in borehole; wifi accelerometer arrays for the high-dynamics monitoring of the buildings; 3D laser scanner; systems for interferometric data.

Data accessibility - open access to archives; data sharing.

Site accessibility services.

Management - Local Unit creation.

All these elements contribute to the configuration of the site that, now, is ready to become a node of the CHeLabS system.

PHASE 3 - Call for proposals

Calls for proposals are periodically launched to collect, evaluate and schedule the future users activities.

PHASE 4 - Performing the scheduled activities proposed by the users.

The site achieves full operability, as shown in Figure 5, hosting the external users activity, such as:

PROJECT 1 - Integration of interferometric data, seismic data, and deformation data.

PROJECT 2 - Digital modelling of monuments by 3D laser scanner and SFM systems (Structure From Motion).

PROJECT 3 - Dynamic analysis of structural decay processes with respect to the maximum deformation of the soil.

PROJECT 4 - Nonlinear dynamics analysis of the soil.

Although a relevant effort should be directed to guarantee the most suitable instrumental equipment onsite, the effectiveness of the model does not depend on the state of the art technologies that, in a specific period of time, are available

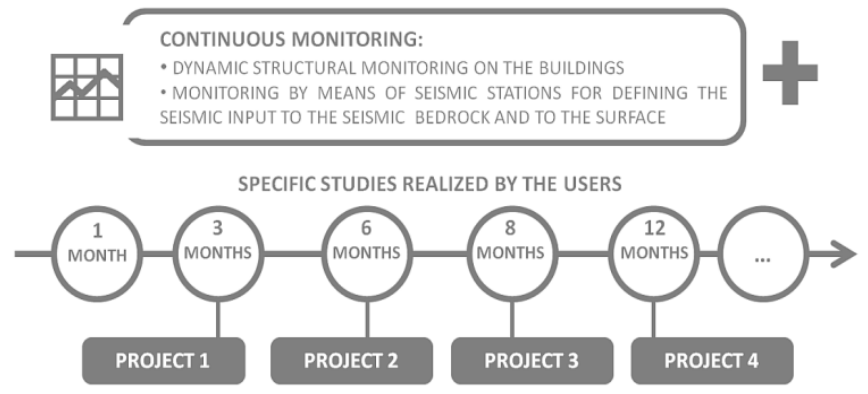

Figure 5. Example of the users activities performed during the full operative phase of a CHeLabS site.

onsite. This means that the implementation of the system shall be continuously renewed with innovative technologies.

The added value of the CHeLabS approach consists in building the more favourable environment that naturally attracts the major experts working on the most challenging issues, in a specific field. Furthermore this system presents a flexibility that, in the long-term, facilitates the achievement of new solutions not necessarily predictable and planned in the implementation phase, thus taking into account that unpredictability characterizing the dynamics of the complex systems evolution.

\section{THE CHELABS PLATFORM}

Today the foundation of the CHeLabS system is set by means of a web-based platform, where the heritage experts together with the entire community are invited to participate to an interactive survey. The principal aims of this survey is to collect and correlate the knowledge needs with the expertise and offer from the other participants, as well as with new and original ideas from the citizens within a vision of public engagement. The participation also gives the opportunity to operate through specific functionalities on an interactive map.

Since this initial phase, the web-based platform of the CHeLabS system tends to represent that shared space facilitating the connectivity among the community, and the exchange of ideas. Figure 6 shows a scheme of the platform structure with its fundamental elements: the community, the survey, and the interactive map.

\subsection{The community}

The potential participants to the survey come from all the public and private organizations and experts that operate in the cultural heritage domain, as well as the citizens.

This wide community has been articulated into six different profiles:

1) territorial organizations, owners, superintendence;

2) conservation operators, heritage scientists;

3) technology operators;

4) schools, high education institutions, universities, research institutes;

5) funding agencies;

6) citizens

Suitable dissemination actions for reaching a statistically significant sample will be undertaken.

\subsection{The survey}

The survey is structured in six questionnaires, that are composed of a section dedicated to gather specific profile 


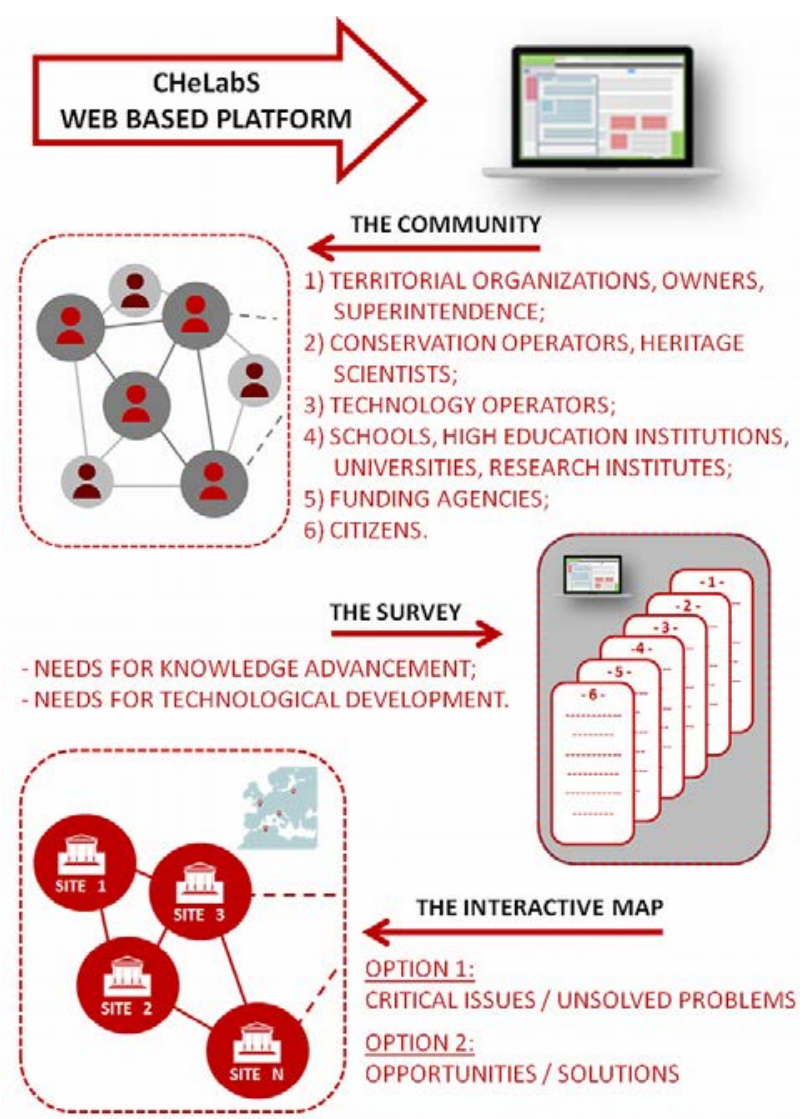

Figure 6. Scheme of the web-based platform of the CHeLabS project, with the main topics regarding the community, the survey, the interactive map.

information, and a common section dedicated to the acquisition of information useful for building need/offer correlations and matching issues between unsolved problems and potential solutions. For each profile the main interest is focused on few questions related to the needs for knowledge advancement and the needs for technological development, indicated by the participants on the base of their professional experience.

The information regarding these needs are transferred to the interactive map, and linked to the heritage sites suggested by the participants, in order to become visible to the whole community.

The contribution from the five profiles, gathering the professionals connected in some ways to the heritage domain, is expected to highlight the current perceived limits demanding for new knowledge, and also the technological trends that could unfold new solutions. On the other hand the sixth profile, gathering the citizens with no specific expertise, has been purposely taken into account for two main reasons: i) mapping the perception of the heritage value, as individuals and as community, together with a sense of belonging; ii) triggering new ideas on expected learning experiences inside a cultural space. This information, provided by this profile, will solicit the experts to move towards a community-driven development.

\subsection{The interactive map}

The participants can operate on an interactive map, and manifest their interest for a heritage site, considered particularly significant for fostering the research. As described above, some information are directly transferred into the map as a contextualization of what is expressed in the questionnaires.
The participants can also add other notes to the dossier of a site according to its specificity; in particular they are invited to play, as in a sort of game, by choosing among two options. One option gives the possibility to briefly express thoughts on critical issues/unsolved problems; the other option gives the possibility to express opportunities/solutions. Thus, starting on a very practical base the participants may virtually meet in a heritage site, share some information and exchange ideas, being involved in a creative discussion. If properly conducted, it is expected that, at least on the most interesting sites, these discussions will evolve according to the chaotically complex dynamics that characterizes the high performance teams, as treated in subsection 2.1.

\section{CONCLUSIONS}

CHeLabS is intended to become a sort of distributed laboratory, attracting competencies and generating excellence, built on the Open Access and Sharing culture. Today, the bottom-up character of the participated survey is inspired by these same principles, inviting the heritage science community together with the citizens to configure a new scenario that will guide the CHeLabS system in its future operating phase.

This paper describes the concept and the current phase of the project, that is moving towards the successive steps of implementation. In the future, the project aims at the integration of a heritage site into the CHeLabS system opening the operative phase. Some efforts have been spent to identify possible pilot sites, and some actions are presently at study. For this task a fundamental step, not deeply discussed in this work, shall regard the configuration of an appropriate partnership including all relevant stakeholders that will be engaged in the management, services and activities on each specific site.

\section{ACKNOWLEDGEMENT}

The initial phase of the CHeLabS project has been funded within the Project SM@RTINFRA - SSHCH (C.N.R. - DSU; MIUR MD n. 973 of 25.11.2013).

The authors wish to acknowledge Sara De Simone for her help in the implementation of the survey, Massimiliano Moscatelli and his research group at the Institute of Environmental Geology and Geoengineering (IGAG CNR) for their relevant help in the definition of the example reported in section 2.4, and Vittorio Loreto (Full Professor at the University of Rome "Sapienza") for his insight into the science of complex systems for a better understanding of the dynamics of innovation. Finally the authors are grateful to the Associate Partners (enterprises and research institutes) that are supporting the CHeLabS initiatives since these first steps.

\section{REFERENCES}

[1] International Charter for the Conservation and Restoration of Monuments and Sites (The Venice Charter). https://www.icomos.org/charters/venice e.pdf, 1964 (accessed 06.03.17).

[2] ICOMOS Statutes. https://www.icomos.org/en/abouticomos/mission-and-vision/statutes-and-policies, 1965 (accessed 06.03.17).

[3] Nara Document on Authenticity. https://www.icomos.org/charters/nara-e.pdf, 1994 (accessed 06.03.17).

[4] Faro Convention. http://www.coe.int/en/web/culture-andheritage/faro-convention, 2005 (accessed 06.03.17). 
[5] Hangzhou Declaration. Placing Culture at the Heart of Sustainable Development Policies. http://www.unesco.org/fileadmin/MULTIMEDIA/HQ/CLT/i mages/FinalHangzhouDeclaration20130517.pdf, 2013 (accessed 06.03.17).

[6] UNESCO and Sustainable Development Goals. http://en.unesco.org/sdgs, 2015 (accessed 06.03.17).

[7] The Florence Declaration on Heritage and Landscape as Human Values.

https://www.icomos.org/images/DOCUMENTS/Secretariat/2 015/GA 2014 results/GA2014 Symposium FlorenceDeclarati on EN final 20150318.pdf, 2014 (accessed 06.03.17).

[8] Manual for Activities directed at Underwater Cultural Heritage. Guidelines to the Annex of the UNESCO 2001 Convention. pp. 22-23 (2013), ISBN 978-92-3-001122-2. http://www.unesco.org/culture/en/underwater/pdf/UCHManual.pdf (accessed 06.03.17).

[9] ESFRI, "European Strategy Report on Research Infrastructures: Roadmap 2016”, ISBN 978-0-9574402-4-1, Mar 2016.

[10] Enhancement of Cultural Heritage through Environmental Planning and Management: CHERPLAN (SEE/0041/4.3/X).
[11] M. Losada, "The complex dynamics of high performance teams", Mathematical and Computational Modelling 30 (1999) pp.179192, 10.1016/S0895-7177(99)00189-2.

[12] V. Loreto, "Unfolding the dynamics of creativity, novelties and innovation", White paper of the Kreyon project, 2015.

[13] V. Loreto, V.D.P. Servedio, S.H. Strogatz and F. Tria "Dynamics on expanding spaces: modeling the emergence of novelties", in: Creativity and Universality in Language. M. Degli Espositi, E.G. Altmann, F. Pachet (editors). Springer International Publishing, 2016, ISBN 978-3-319-24403-7, pp.59-83.

[14] O. Caridi-Zahavi, A. Carmeli, and O. Arazy, "The influence of CEOs' visionary innovation leadership on the performance of high-technology ventures: the mediating roles of connectivity and knowledge integration", Journal of Product Innovation Management 33, Iss.3 (2016) pp.356-376, 10.1111/jpim.12275.

[15] A. Carmeli, P.B. Paulus, "CEO ideational facilitation leadership and team creativity: the mediating role of knowledge sharing", The Journal of Creative Behavior 49, Iss.1 (2014) pp. 53-75, 10.1002/jocb.59.

[16] UNESCO "Open Access for Researchers - Module 2 Concepts of Openness and Open Access", 2015, ISBN 978-92-3-100079-9. 